\title{
Safety of Unilateral Endovascular Occlusion of the Cervical Segment of the Vertebral Artery without Antecedent Balloon Test Occlusion
}

G.H. Zoarski and R. Seth

\begin{abstract}
BACKGROUND AND PURPOSE: Antecedent balloon test occlusion is often performed prior to vertebral artery sacrifice, but there is limited data to suggest this adds a significant clinical benefit, especially in the setting of trauma. Furthermore, balloon test occlusion can be time-consuming, add to the technical complexity of the procedure, and increase the overall cost of treatment. The purpose of this study was to determine the safety of unilateral vertebral artery occlusion without antecedent balloon test occlusion as part of the treatment regimen in patients with traumatic vertebral artery dissection, cervical tumor, or intracranial aneurysm.
\end{abstract}

MATERIALS AND METHODS: The medical records and imaging studies of 59 patients in whom unilateral endovascular cervical vertebral artery occlusion was performed were retrospectively reviewed. Procedure-related stroke was defined as imaging evidence of acute infarct in the vascular territories supplied by the occluded vertebral artery or new focal neurologic deficit developing in the first 30 days after vertebral artery occlusion attributable to infarction in the posterior circulation.

RESULTS: Fifty-nine patients underwent unilateral endovascular cervical vertebral artery occlusion to prevent potential thromboembolic complications of vertebral artery injury, for treatment of intracranial aneurysms, or for presurgical embolization of a cervical vertebral tumor. Unilateral occlusion was performed when endovascular reconstruction was considered impossible or deemed more risky than deconstruction. Fifty-eight of the 59 patients underwent vertebral artery occlusion without antecedent balloon test occlusion. None of the 59 patients had clinical or imaging evidence of a postprocedural infarct.

CONCLUSIONS: In this series, endovascular occlusion of a cervical segment of 1 vertebral artery was safely performed without antecedent balloon test occlusion. As long as both vertebral arteries were patent and converged at the vertebrobasilar junction, there was anatomic potential for retrograde filling of the distal intracranial vertebral artery to the level of the posterior inferior cerebellar artery origin, and there was no major vascular supply to the spinal cord arising from the target segment of the affected vessel. Dominant and nondominant vertebral arteries were safely occluded, and no infarcts were attributed to the treatment.

ABBREVIATIONS: BTO = balloon test occlusion; VA = vertebral artery

i $\mathrm{n}$ most individuals, the basilar artery benefits from dual arterial inflow from the vertebral arteries (VAs). A rich cervical collateral network may further augment posterior circulation flow to a variable degree. This anatomic configuration is extremely effective in protecting the brain stem from ischemia in unilateral vertebral artery injury and allows therapeutic occlusion of the cervical segment of one of the vertebral arteries in most patients.

Received April 30, 2013; accepted after revision October 11.

From the Department of Neurointerventional Surgery (G.H.Z.), Christiana Care Health System, Newark, Delaware; and Department of Neuroradiology (R.S.), Radiology Associates of North Texas, Fort Worth, Texas.

Please address correspondence to Gregg H. Zoarski, MD, Neurointerventional Surgery, Christiana Care Health System, 4755 Ogletown-Stanton Rd, Suite 1E10, Newark, DE 19718; e-mail: gzoarski@gmail.com

http://dx.doi.org/10.3174/ajnr.A3885
Asymptomatic occlusion of the cervical vertebral artery, particularly at the level of its origin from the subclavian artery, is not unusual in patients with severe atherosclerosis. In many such cases, segmental cervical collaterals effectively reconstitute the vertebral artery below the level of the skull base. These collateral vessels often arise from muscular branches of the vertebral artery, the ascending cervical branch of the thyrocervical trunk, or the deep cervical branch of the costocervical trunk (Fig 1). Furthermore, segmental spinal arteries and the normal confluence of both vertebral arteries provide a rich collateral supply to the posterior fossa.

Due to the theoretic potential for significant morbidity from posterior circulation ischemia, intentional occlusion of even a severely compromised vertebral artery is undertaken with abundant concern for patient safety. There is little evidence that antecedent balloon test occlusion (BTO) of 1 vertebral artery is nec- 


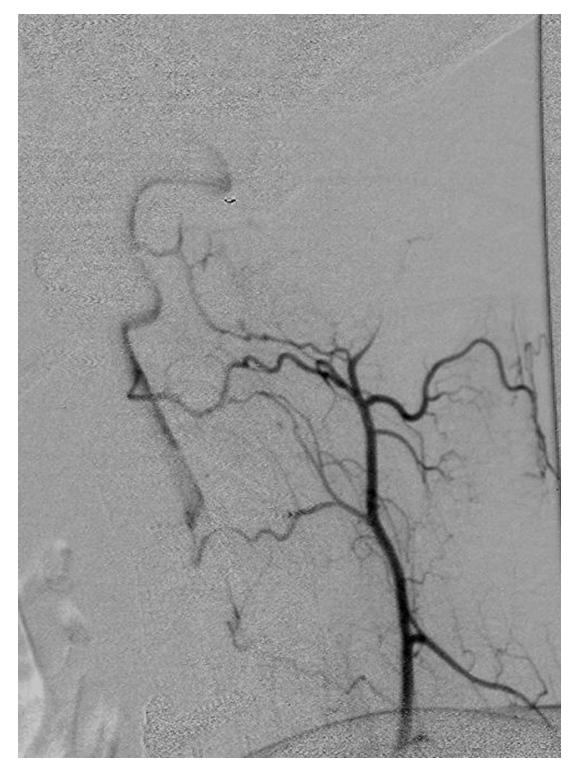

FIG 1. A 24-year-old man with C6-C7 fracture dislocation, spinal cord injury, and traumatic occlusion of the left vertebral artery following a motor vehicle collision. DSA of the left costocervical trunk demonstrates distal reconstitution of the occluded midcervical left VA from the posterior deep cervical branch of the costocervical trunk. The left VA is occluded proximally.

essary, or even reliable, in predicting ischemic deficits. ${ }^{1,2}$ Additionally, balloon test occlusion can be time-consuming, add to the technical complexity of the procedure, and increase the overall cost of treatment. There are limited data to suggest that performing a BTO before vertebral artery sacrifice adds clinical benefit, especially in the setting of trauma, where patients are often intubated and sedated. To our knowledge, a large series of patients undergoing unilateral endovascular vertebral artery occlusion without antecedent BTO has not been previously published.

In a retrospective review of 28 treated dissecting vertebrobasilar aneurysms by Rabinov et $\mathrm{al}^{3} 7$ of the 28 treated patients underwent an antecedent BTO. The authors speculated that "BTO may be of benefit to determine if the collateral circulation is limited anatomically or compromised by vasospasm," but provided no data to suggest that BTO changed management in any patients or led to improved outcome in the group that underwent BTO. A separate surgical report of 15 patients describes vertebral artery ligation before cervical tumor resection without antecedent BTO, performed only if the contralateral vertebral artery was larger or equal in diameter to the sacrificed vessel. No cases of cerebral or spinal cord ischemia were reported in this study. ${ }^{4}$ Given this information and our own personal experience, we believe that unilateral therapeutic occlusion of a cervical vertebral artery is safe as long as the contralateral vertebral artery is patent to the level of the vertebrobasilar junction and there is no angiographic evidence of vascular supply to the spinal cord arising from the target segment of the affected vessel.

The purpose of this study was to review our experience in therapeutic occlusion of the cervical vertebral artery in the setting of traumatic injury, as part of distal vertebrobasilar aneurysm treatment, or to facilitate surgical resection of adjacent cervical tumors. Our rationale for therapeutic occlusion in the setting of
VA injury was based on the belief that sudden recanalization of an occluded vertebral artery could result in thromboembolism and consequent stroke. However, the actual likelihood and incidence of such embolic events are unknown, and intentional vertebral artery occlusion should, therefore, only be performed if the risk of the procedure is determined to be small. Furthermore, we performed vertebral artery occlusion only when endovascular reconstruction was considered impossible or deemed more risky than deconstruction.

\section{MATERIALS AND METHODS}

\section{Patients}

The clinical records and imaging studies of 59 patients treated with unilateral endovascular vertebral artery occlusion between 1991 and 2006 were retrospectively reviewed following approval by our institutional review board. Patient clinical information was logged into a data base, and conventional and CT angiographic imaging was reviewed. Variables recorded included clinical presentation, diameter of the bilateral vertebral arteries, side of vertebral artery occlusion, endovascular techniques used, and the angiographic and clinical outcomes of treatment.

\section{Occlusion Techniques}

In most cases $(n=55)$, coils were used to achieve vertebral artery occlusion. Balloons, silk suture, or a combination of these devices were used for occlusion in the remaining 4 patients.

A successful BTO was performed before permanent occlusion in a single patient. In the remaining 58 patients, permanent vertebral artery occlusion was performed without BTO. The affected vertebral artery was only occluded when confluence of both vertebral arteries at the level of the vertebrobasilar junction was confirmed and the anatomic potential for retrograde filling of the vertebral artery down to the level of the posterior inferior cerebellar artery origin on the affected side was firmly established (Fig $2 B)$. The potential for PICA filling was defined either by identification of antegrade opacification of the bilateral V4 segments and basilar artery, with or without opacification of the PICA on the affected side, or by retrograde opacification of the entire V4 segment of the affected vertebral artery on contralateral VA injection. Opacification of the PICA on the affected side was not necessary because opacification of the V4 segment of the affected vertebral artery suggested that the PICA would fill after ipsilateral vertebral artery occlusion.

Dominance of the affected vertebral artery or the presence or size of the posterior communicating arteries did not affect the decision to perform the occlusion. In many cases, the dominant vertebral artery was already completely or near completely occluded, or the need for occlusion of the dominant vertebral artery was clinically imperative. In cases of complete occlusion of the vertebral artery secondary to traumatic injury, coil embolization of the occluded stump (Fig 2C) was performed to prevent emboli in case of spontaneous recanalization.

In 55 patients, the vertebral artery was occluded only proximal to the site of injury. Arteriovenous fistulas were identified in 4 patients, and occlusion was performed both proximal and distal to the site of injury to eliminate arteriovenous flow in these 4 patients.

AJNR Am J Neuroradiol 35:856-61 May 2014 www.ajnr.org 

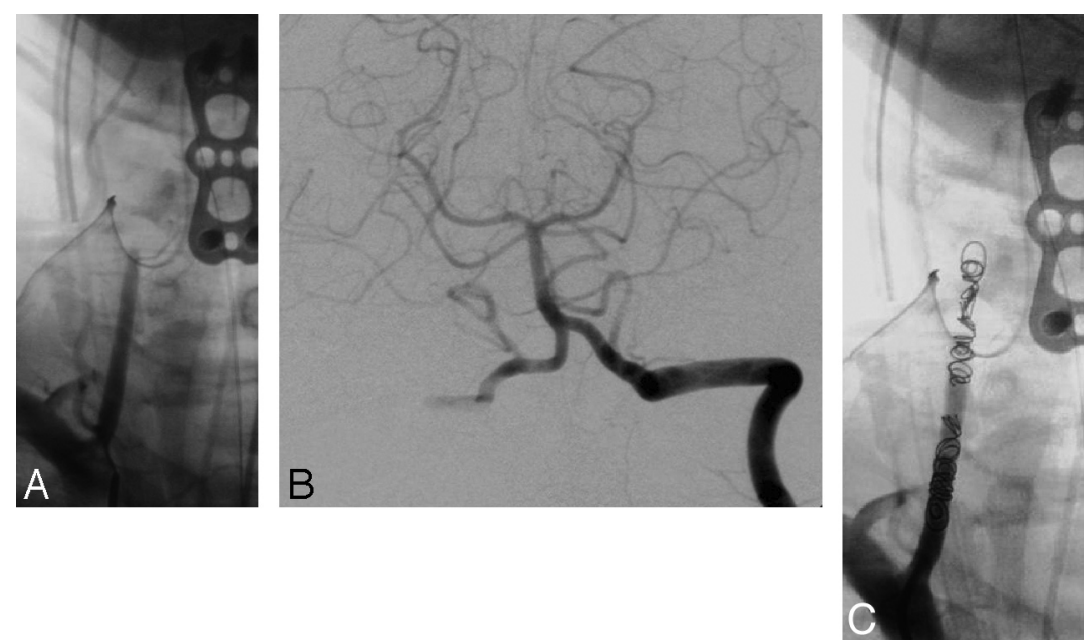

artery occlusion was performed before surgical resection of tumors of the cervical vertebrae.

Fifty-eight (98.3\%) of the 59 patients underwent vertebral artery occlusion without antecedent BTO. In 33 (55.9\%) of 59 cases, the left vertebral artery was treated with endovascular occlusion; the right vertebral artery was treated in the remaining 26 (44.1\%). Vascular measurements of the cervical vertebral artery were made on conventional angiographic or CT angiographic images in 35 (59.3\%) of the 59 cases. Of these 35 cases, the dominant or codominant vertebral artery was treated in $29(82.9 \%)$ patients, while the

FIG 2. A 35-year-old man with a C5 burst fracture, right vertebral artery occlusion, and spinal cord injury following a motor vehicle collision. A, Anteroposterior digital subtraction angiography of the right vertebral artery shows occlusion at the level of $C 5$ before endovascular treatment. $B$, Anteroposterior DSA of the intracranial left vertebral artery demonstrates backfilling of the occluded right vertebral artery down to the level of the right posterior inferior cerebellar artery. C, Anteroposterior angiographic image demonstrates occlusion of the right vertebral artery stump secured with fibered coils to avoid emboli in spontaneous recanalization. nondominant vertebral artery was treated in $6(17.1 \%)$.

Complete angiographic occlusion of the target segment of the treated vertebral artery was achieved in all cases. Contralateral vertebral artery angiography after vessel occlusion demonstrated retrograde

When surgical comorbidities permitted, postprocedural systemic anticoagulation was administered to achieve a partial thromboplastin time of 40-50 seconds; and if clinically feasible, patients received antithrombotic treatment with aspirin, 81 or $325 \mathrm{mg}$ for 90 days following endovascular occlusion.

\section{Follow-Up}

Follow-up CT or MR imaging of the brain was performed within 48 hours after treatment in all patients. Procedure-related stroke was defined as CT or MR imaging evidence of an acute infarct in the cerebral, cerebellar, or spinal cord vascular territories supplied by the occluded vertebral artery or a new focal neurologic deficit developing in the first 30 days after vertebral artery occlusion attributable to infarction in the posterior circulation.

\section{RESULTS}

Fifty-nine patients treated with endovascular unilateral vertebral artery occlusion between 1991 and 2006 were identified. Forty-four (74.6\%) patients were male, and 15 (25.4\%) were female. Patient age ranged from 14 to 73 years, with a mean age of 35.5 years.

Twenty-nine of 59 patients were treated due to traumatic vascular injury of the vertebral artery secondary to deceleration injury sustained during a motor vehicle collision or fall. Twenty-one patients were treated for penetrating trauma, most commonly gunshot wounds or stab injuries. Four patients were treated for vertebral artery injury during a surgical procedure or line placement. Unilateral vertebral artery occlusion was undertaken in the setting of vertebral artery injury with flow-limiting stenosis $(>70 \%)$ in patients who were not candidates for traditional therapy of systemic anticoagulation or in cases in which intraluminal thrombus created concern for spontaneous embolization. In all cases, the contralateral vertebral artery was patent and without evidence of intimal injury. In 2 patients, proximal occlusion of a unilateral cervical vertebral artery was performed as part of the treatment plan for a distal aneurysm. In 3 cases, cervical vertebral opacification of the intracranial V4 segment of the occluded vertebral artery in all cases. Of the 59 patients treated, none had a stroke attributed to intentional vertebral artery occlusion, and there were no instances of delayed ischemic events attributable to stump emboli or target vessel recanalization.

\section{DISCUSSION \\ Safety of Unilateral Vertebral Artery Occlusion}

Surgical ligation and endovascular deconstruction or segmental occlusion of the vertebral artery has long been used to facilitate resection of cervical spinal tumors and treat difficult intracranial aneurysms. ${ }^{1,4-6}$ In some cases, surgical or endovascular vessel sacrifice can obviate complex skull base surgery. Dissecting aneurysms of the intracranial vertebral artery are particularly difficult to reconstruct surgically and have been treated successfully with proximal vertebral artery or endovascular segmental occlusion of the affected vessel..$^{7-10}$ Untreated, these lesions carry an inherent high risk of subarachnoid hemorrhage. Furthermore, untreated ruptured dissecting aneurysms have a high propensity for rehemorrhage, with rates ranging from $30 \%$ to $70 \% .^{11,12}$

Previous authors have demonstrated the feasibility and success of surgical or endovascular occlusion of the intracranial vertebral artery with low rates of ischemic or thromboembolic complications. ${ }^{4,13}$ The favorable safety profile demonstrated in our study of 59 patients is consistent with these studies as well as a recent report by Kansagra et al, ${ }^{14}$ in which 100 traumatic arterial cervicocerebral vascular injuries were endovascularly treated with a low rate of immediate or delayed neurovascular complications.

The potential adverse consequences of ligation of the vertebral artery are not insignificant. Symptoms of vertebrobasilar stroke include vertigo, nausea, vomiting, headache, visual field deficit, diplopia, pupillary abnormalities, ophthalmoplegia, agnosia, and alexia without agraphia. More significant deficits such as facial paralysis, dysarthria, sudden deafness, lower cranial nerve defi- 

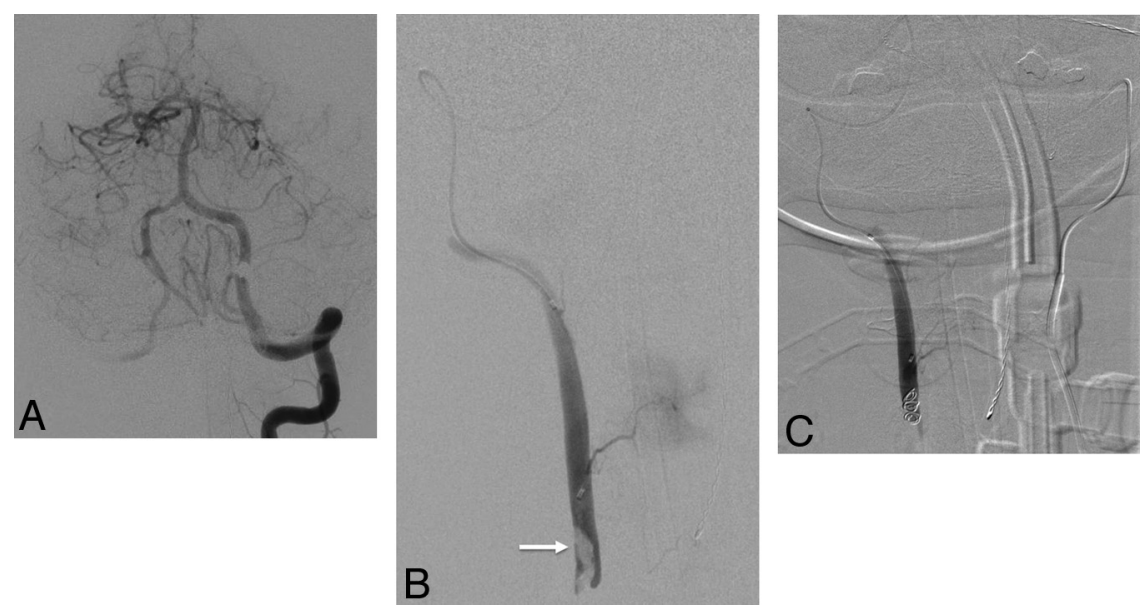

FIG 3. A 45-year-old man with fracture and diastasis of the right $\mathrm{C} 4-\mathrm{C} 5$ facet joint, with fracture extending through the right $\mathrm{C} 5$ transverse foramen, following a motorcycle collision. A, Anteropostior DSA of the left VA shows retrograde flow down the distal right VA due to proximal occlusion. $B, A$ microcatheter was passed from the left VA to the vertebrobasilar junction, and retrograde microcatheterization of the right VA was performed. Hand-injection DSA demonstrates thrombus (arrow) in the lumen. C, Anteroposterior DSA with injection of the microcatheter following placement of coils above the thrombus to prevent embolization.

cits, altered awareness, Wallenberg syndrome, or Foville syndrome may also occur. ${ }^{15,16}$

\section{Endovascular Occlusion}

Demonstration of the confluence of both vertebral arteries at the level of the vertebral junction and the anatomic potential for retrograde filling of the PICA on the affected side was paramount in establishing the feasibility of unilateral therapeutic vertebral artery occlusion. Dominance of the affected vertebral artery did not affect our decision to perform the occlusion when the need for occlusion of the affected vertebral artery was clinically imperative. When the dominant vertebral artery was already occluded or near-occluded, intact neurologic examination findings demonstrated that the patient was already tolerating a functional test occlusion. In fact, it was our observation of several neurologically intact patients who presented with acute traumatic occlusion of the dominant vertebral artery that led us to believe that therapeutic occlusion of even the dominant vertebral artery could be performed safely on the basis of anatomic criteria without antecedent balloon test occlusion.

Preservation of flow into the PICA on the affected side is an essential component of therapy. Unlike reports describing endovascular treatment of dissecting intracranial vertebral artery aneurysms, most therapeutic occlusions in our series were performed for vertebral artery pathology located several cervical vertebral segments proximal to the origin of the PICA. In some cases, collaterals distal to the site of pathology already reconstituted the distal cervical vertebral artery and filled the PICA in an antegrade fashion before proximal or segmental vertebral artery occlusion. Without such cervical collaterals, the ipsilateral PICA was typically opacified by retrograde flow from the vertebrobasilar junction even before vertebral artery occlusion.

In the setting of a severe vascular injury, such as vertebral artery fistula or frank extravasation, proximal occlusion of the vertebral artery alone is insufficient treatment because the lesion will continue to derive flow from the patent distal cervical verte- bral artery in retrograde fashion. Occlusion both proximal and distal to the lesion must be secured in such cases. In many instances, it is possible to advance a catheter or microcatheter beyond the level of the lesion in antegrade fashion to perform an initial distal occlusion. Proceeding in retrograde fashion, one can use coils, glue, particles, or balloons to occlude the vertebral artery at and below the level of the pathology. ${ }^{17}$ In the case of a fistula or frank extravasation, embolic materials may also be placed outside the arterial lumen, as long as sufficient care is taken to avoid venous migration. When the proximal segment of the affected vertebral artery is already completely or near-completely occluded, coils can be placed distal to the level of injury by navigating a microcatheter through the contralateral vertebral artery, across the vertebrobasilar junction, and retrograde to the level of vascular injury (Fig 3). ${ }^{18}$

Regardless of the level or degree of vertebral artery pathology, the technique of occlusion must be designed to maintain the patency of the PICA origin and the vertebral artery contribution to the anterior spinal artery on the treatment side. Reconstruction of the injured segment should always be considered before vertebral artery occlusion, especially when the injured vessel is markedly dominant. However, neurologic sequelae from unilateral vertebral artery occlusion are unlikely in the presence of a normal contralateral vessel and in the absence of angiographic demonstration of branches to the spinal cord. ${ }^{19}$

\section{Vertebral Artery Balloon Test Occlusion}

While it is tempting to believe that balloon test occlusion of a vertebral artery may predict the possibility of posterior circulation stroke after permanent vertebral artery occlusion, the utility of vertebral artery balloon test occlusion has only been reported in small series, ${ }^{1,2}$ none of which attest to the reliability or validity of vertebral artery test occlusion in predicting subsequent stroke after permanent vertebral artery occlusion. While it is clearly evident that a failed test occlusion predicts a poor outcome after permanent occlusion, there are no quantitative data to suggest that the converse is true and that passing a BTO before permanent vertebral artery occlusion diminishes the risk of subsequent posterior circulation stroke. Nevertheless, several authors prudently recommend that balloon test occlusion be performed before permanent occlusion in certain circumstances, including a contralateral vertebral artery that does not communicate with the vertebrobasilar junction, the presence of a markedly hypoplastic contralateral vertebral artery, or anticipated occlusion of a strongly dominant vertebral artery.

In a series of 23 patients with dissecting aneurysms of the vertebral artery, Albuquerque et a ${ }^{13}$ suggested that "dominance of the affected artery complicates the treatment paradigm. In such cases, balloon test occlusion is helpful in assessing the risk of 
stroke after vessel sacrifice." Only anecdotal references are provided to support this recommendation, and balloon test occlusion was only performed in a single patient in whom the affected artery was dominant. Hoshino et $\mathrm{al}^{4}$ reported a series of 15 patients in whom no ischemic complications were demonstrated following ligation of the nondominant or codominant cervical vertebral artery. Although the authors suggested that angiographic test occlusion of the involved vertebral artery should be performed before permanent occlusion, only anecdotal support was provided for this recommendation. In their series, BTO was performed in a single patient who had a single vertebral artery, and the patient predictably became symptomatic during the BTO procedure. $^{4}$

Luo et $\mathrm{al}^{20}$ treated 10 patients with spontaneously ruptured vertebral dissecting aneurysms and proposed that sudden occlusion of the vertebral artery carries a risk of ischemia "particularly in patients who have a hypoplastic contralateral vertebral artery," and that "balloon occlusion test (BOT) is essential to determine the adequacy of collateral circulation from the contralateral vertebral artery or posterior communicating arteries." This determination was made despite any substantial evidence; however, the authors concede that "although BOT in the vertebrobasilar system is less effective in predicting future ischemia, it can indicate the extent of collateral circulation in the vertebrobasilar system." Similarly, Rabinov et $\mathrm{al}^{3}$ described 26 patients with intracranial vertebrobasilar dissecting aneurysms who were treated by endovascular techniques. Although only 7 patients were evaluated with preprocedural BTO and no patients were reported to have failed BTO, the authors concluded that "BTO may be of benefit to determine if the collateral circulation is limited anatomically or compromised by vasospasm. ${ }^{3}$ Despite the cautionary statements found in these articles, there are no published reports, to our knowledge, of a patient failing to tolerate vertebral BTO when the contralateral vertebral artery was documented to be patent to the level of the vertebrobasilar junction.

On the other hand, the literature demonstrates that patients who tolerate BTO of a unilateral vertebral artery may nevertheless experience ischemia and infarction after permanent vertebral artery occlusion. Sorteberg et $\mathrm{al}^{2}$ have demonstrated the use of transcranial Doppler sonography to evaluate blood velocity and blood flow direction in the P1 segments during BTO to rapidly predict hemodynamic outcome at a risk comparable with that of conventional neuroangiography. Reversal of flow in the P1 segment following balloon inflation suggested that the patient was likely to tolerate permanent occlusion of the affected vertebral artery. Although all 7 patients who underwent unilateral VA BTO with transcranial Doppler in their study passed the test occlusion, a single patient developed Brown-Sequard syndrome due to medullary infarction after subsequent permanent occlusion of the intracranial VA just proximal to the origin of the PICA. The authors stated that spinal medullary branches may be too small to visualize during angiography and BTO cannot reliably exclude the risk of occlusion of a spinal medullary branch. ${ }^{2}$ However, infarction in this case occurred subsequent to occlusion of the intracranial segment of the vertebral artery, where the potential for segmental cervical muscular collaterals to reconstitute the distal vertebral artery is absent. Delayed ischemic events, despite uneventful bal- loon test occlusion, have been described by other authors and may be related to hypoperfusion not predicted by BTO. ${ }^{1}$

Overall, BTO does not enhance the safety of subsequent occlusion of a unilateral vertebral artery, regardless of the reason or vessel size, if there is anatomic potential for filling of the ipsilateral PICA and there is no major vascular supply to the spinal cord arising from the target segment of the affected vessel.

\section{Alternative Treatment Strategies/Conservative Therapy}

Despite the lack of complications in our series, traditional alternatives to vertebral artery occlusion, such as systemic anticoagulation or antiplatelet therapy, are adequate treatment in many patients. ${ }^{21-23}$ Biffl et $\mathrm{al}^{24}$ reviewed the presentation, treatment, and outcome of 38 patients presenting with 47 blunt vertebral artery injuries during a 3.5 -year period. The incidence of posterior circulation stroke was $24 \%$, and death attributable to vertebral artery injury was $8 \%$. They determined that systemic heparin therapy was effective in preventing stroke, neurologic deterioration, and progression to higher injury grade in patients both with and without established stroke. Additionally, dominance of the affected vertebral artery, the presence of bilateral injuries, or the initial injury grade did not appear to influence the incidence of stroke. While it has been speculated that nonocclusive injuries may be more dangerous than complete occlusions, this possibility was also not supported by their findings. The authors cautioned that anticoagulation may have resulted in complications, including hemorrhagic infarction; however, the overall benefit of systemic heparin therapy outweighed these potential complications. At our own institution, we evolved toward earlier and more frequent use of oral antiplatelet agents in patients with traumatic vascular injury in the absence of embolic sequelae. However, anticoagulation and antiplatelet therapies are not entirely benign and may still leave the patient at risk for thromboembolic events. Serious or fatal bleeding may occur, especially with multisystem trauma, and may be difficult or impossible to control.

\section{CONCLUSIONS}

Endovascular occlusion of the cervical segment of 1 vertebral artery can be safely performed without antecedent BTO as long as both vertebral arteries are patent and converge at the vertebrobasilar junction, there is anatomic potential for retrograde filling of the distal intracranial vertebral artery down to the level of the PICA origin, and there is no vascular supply to the spinal cord arising from the target segment of the affected vessel. Nondominant and dominant vertebral arteries were safely occluded following careful angiographic evaluation of posterior circulation anatomy in our series, and no infarcts were attributed to the treatment. We routinely perform unilateral cervical vertebral artery occlusion without prior balloon test occlusion in these circumstances, regardless of vertebral artery dominance, when endovascular reconstruction is considered impossible or deemed more risky than deconstruction.

\section{REFERENCES}

1. Graves VB, Perl J 2nd, Strother CM, et al. Endovascular occlusion of the carotid or vertebral artery with temporary proximal flow 
arrest and microcoils: clinical results. AJNR Am J Neuroradiol 1997;18:1201-06

2. Sorteberg A, Bakke SJ, Boysen M, et al. Angiographic balloon test occlusion and therapeutic sacrifice of major arteries to the brain. Neurosurgery 2008;63:651-60

3. Rabinov JD, Hellinger FR, Morris PP, et al. Endovascular management of vertebrobasilar dissecting aneurysms. AJNR Am J Neuroradiol 2003;24:1421-28

4. Hoshino Y, Kurokawa T, Nakamura K, et al. A report on the safety of unilateral vertebral artery ligation during cervical spine surgery. Spine 1996;21:1454-57

5. Drake CG. Ligation of the vertebral (unilateral or bilateral) or basilar artery in the treatment of large intracranial aneurysms. J Neurosurg 1975;43:255-74

6. Barr JD, Lemley TJ. Endovascular arterial occlusion accomplished using microcoils deployed with and without proximal flow arrest: results in 19 patients. AJNR Am J Neuroradiol 1999;20:1452-56

7. Tanaka K, Waga S, Kojima T, et al. Non-traumatic dissecting aneurysms of the intracranial vertebral artery: report of six cases. Acta Neurochir (Wien) 1989;100:62-66

8. Iihara K, Sakai N, Murao K, et al. Dissecting aneurysms of the vertebral artery: a management strategy. J Neurosurg 2002;97:259-67

9. Leibowitz R, Do HM, Marcellus ML, et al. Parent vessel occlusion for vertebrobasilar fusiform and dissecting aneurysms. AJNR Am J Neuroradiol 2003;24:902-07

10. Halbach VV, Higashida RT, Dowd CF, et al. Endovascular treatment of vertebral artery dissections and pseudoaneurysms. J Neurosurg 1993;79:183-91

11. Aoki N, Sakai T. Rebleeding from intracranial dissecting aneurysm in the vertebral artery. Stroke 1990;21:1628-31

12. Mizutani T, Aruga T, Kirino T, et al. Recurrent subarachnoid hemorrhage from untreated ruptured vertebrobasilar dissecting aneurysms. Neurosurgery 1995;36:905-11, discussion 912-13
13. Albuquerque FC, Fiorella DJ, Han PP, et al. Endovascular management of intracranial vertebral artery dissecting aneurysms. Neurosurg Focus 2005; 18:E3

14. Kansagra AP, Cooke DL, English JD, et al. Current trends in endovascular management of traumatic cerebrovascular injury. $\mathrm{J} \mathrm{Neu-}$ rointerv Surg 2014;6:47-50

15. Shintani A, Zervas NT. Consequence of ligation of the vertebral artery. J Neurosurg 1972;36:447-50

16. Nakaso K, Nakayasu H, Isoe K, et al. A case of dissecting aneurysm of the basilar artery presenting as a superior pons type of Foville syndrome [in Japanese]. Rinsho Shinkeigaku 1995;35:1040-43

17. Herrera DA, Vargas SA, Dublin AB. Endovascular treatment of traumatic injuries of the vertebral artery. AJNR Am J Neuroradiol 2008;29:1585-89

18. Ji KY, Ahn JY, Song WS, et al. Endovascular treatment for a penetrating vertebral artery injury. J Korean Neurosurg Soc 2002;32:578-81

19. Golueke P, Sclafani S, Phillips T, et al. Vertebral artery injury: diagnosis and management. J Trauma 1987;27:856-65

20. Luo CB, Chang CY, Teng MM, et al. Endovascular treatment of ruptured vertebral dissecting aneurysms with electrodetachable coils. J Chin Med Assoc 2005;68:578 - 84

21. Cimini N, D'Andrea P, Gentile M, et al. Cervical artery dissection: a 5-year prospective study in the Belluno district. Eur Neurol 2004;52:207-10

22. Cervical Artery Dissection in Stroke Study Trial Investigators. Antiplatelet therapy vs. anticoagulation in cervical artery dissection: rationale and design of the Cervical Artery Dissection in Stroke Study (CADISS). Int J Stroke 2007;2:292-96

23. Menon R, Kerry S, Norris JW, et al. Treatment of cervical artery dissection: a systematic review and meta-analysis. J Neurol Neurosurg Psychiatry 2008;79:1122-27

24. Biffl WL, Moore EE, Elliott JP, et al. The devastating potential of blunt vertebral arterial injuries. Ann Surg 2000;231:672-81 